\title{
II. (CXCIV.) Os lunatum carpi mit einem Anhange am dorsalen Ende. Lunatum bipartitum 3. Art.
}

(Hierzu Taf. VIII. Fig. 3-4.)

Die andere Art von Ueberzahl der Knochen im menschlichen Carpus ist durch Zerfallen der gewöhnlichen Carpalknochen in 2 bis 3 stücke bedingt.

\section{a. In 2 Stücke.}

Das Naviculare, welches in ein $\mathrm{N}$. secundarium radiale et ulnare zerfallen ist, - Naviculare bipartitum - babe ich zuerst beobachtet (1866 etc.). (Sein Vorkommen wurde später von mir und Anderen bestätigt.) Das Lunatum sah in ein vorderes und hinteres Stück getheilt Smith (1847), in ein L. secundarium dorsale und in ein ganz kleines L. s. volare geschieden zuerst ich (1870). Die Spur einer Partition des Triquetrum in ein T. s. dorsale et volare beobachtete ich (1870). Das Maltangulum majus mit einer selbständig gewordenen Epiphyse versehen traf ich $2 \mathrm{Mal}$ an (1875). Ich beobachtete mit Max Flesch, und unabhängig von diesem, das Multangulum minus getheilt in ein M. m. secundarium dorsale et volare - Multangulum minus bipartitum - (1879). [Sein Vorkommen wurde bestätigt durch Turner (1883)].

\section{b. In 3 Stücke.}

Das Capitatum in ein C. secundarium superius, C. s. radiale et C. s. ulnare getheilt - Capitatum tripartitum - sah ich (1870). Zusammen mit einem wirklichen Naviculare bipartitum beobachtete ich ein Knochenstïckchen (1877), in welchem ich jetzt das Centrale zu vermuthen mich für berechtigt halte; auch traf ich das wirkliche Centrale in einem gewissen Grade seiner Verwachsung mit einem Naviculare, an welchem ich Zeichen vorfand, die ich für die Spuren dagewesener Partition in einer früheren Lebenszeit nehmen zu dürfen meine (1883) - also Naviculare tripartitum. -

Die 1. Variante eines Lunatum bipartitum sah, wie gesagt, R. W. Smith ${ }^{1}$ ) an einer, auf die Volarfläche des Vorderarm-

1) Treatise on fractures and dislocations. Dublin 1847. p.252. (Steht mir nicht zur Verfügung, aber bei E. Gurlt, Beiträge z. vergleich.- 
knochens angeboren luxirten Hand eines jungen Frauenzimmers. Es bestand aus 2 vollkommen getrennten Portionen, einer vorderen und einer hinteren. Die hintere Portion, welche die überzählige zu sein schien, hatte 5 Gelenkflächen zur Articulation mit dem Naviculare, Triquetrum, Capitatum, der vorderen Portion des Lunatum und dem Radius.

Die 2. Variante eines Lunatum bipartitum, bei welcher die stumpfe volare untere Ecke des Lunatum ein von diesem Knochen separirtes Ossiculum darstellt, also das Lunatum in ein sehr grosses L. secundarium dorsale und in ein sehr kleines L. s. volare getheilt ist, habe ich zuerst, und zwar 1869 an dem Lunatum der rechten Hand mit Weichtheilen von einem Manne und 1870 an einem macerirten linken Lunatum beobachtet').

In beiden Fällen wurde im L. s. volare eine an der volaren unteren Ecke des knorpligen Lunatum aus einem besonderen Knochenkerne entwickelte Epiphyse erkannt, deren Synchondrose in einem Falle zu einer Art Gelenkkapsel sich entwickelt hatte, in dem anderen Falle aber später verknöchert war.

Im ersten Falle hatte das L. s. volare die Gestalt eines Viertelsegmentes eines quer gelagerten, kleinen, ovalen Körpers, welcher durch eine straffe Gelenkkapsel und einige Fäden mit dem L. s. dorsale vereinigt war und mit einer Gelenkfläche, welche die radiale Facette der Gelenkfläche der S. digitalis des L. s. dorsale vergrösserte, am Capitatum articulirte. Im zweiten Falle hatte das L. s. volare eine ïhnliche Gestalt oder die einer dreieckigen oder halbovalen Platte, die mit dem L. s. dorsale, wegen bereits eingetretener Verknöcherung in der Synchondrose, nicht mehr gelenkig verbunden war und mit einer überknorpelt gewesenen S. digitalis, die in der Verlängerung der radialen

path. Anatomie d. Gelenkkrankheiten. Berlin 1853, S. 364 , dann bei Humphry, A treatise on the human skeleton. London 1858. $8^{\circ}$. p. 397. Note 2.)

1) a) Beiträge zu den secundären Handwurzelknochen des Menschen. Arch. f. Anat., Physiol. u. wissensch. Medicin. Leipzig 1870. S. 493-494. Fig. 4-5. - b) Beobachtung eines ursprünglich in zwei Lunata secundaria zerfallen gewesenen Lunatum der linken Hand eines Erwachsenen. Bull. de l'Acad. Imp. des sc. de St. Petersbourg. Tom. XV. 1870. Col. $449-451$. 


\section{1}

Facette der S. digitalis des Lunatum s. dorsale liegt, wieder mit dem Capitatum articulirt haben musste.

Den angegebenen Arten von Partition des Lunatum kann ich eine neue 3. Art hinzufügen, bei der das Lunatum in ein ganz grosses L. secundarium volare und in ganz kleines L. s. dorsale zerfallen ist. Einen Fall dieser Art beobachtete ich am 9. November 1880 an der rechten Hand mit Weichtheilen von einem männlichen Subjecte (Fig. 3-4 No. 1). Ich fand den Fall erst bei der Durchmusterung von 2501 (1242 rechtsseitigen und 1259 linksseitigen) Händen an der 1242. rechtsseitigen Hand, was die Seltenheit des Vorkommens beweist.

An dem aufbewahrten und vor mir liegenden Präparate ist Folgendes zu bemerken:

Das Lunatum (No. 1) gehört zur schmäleren Art, hat deshalb am Knorpel seiner Superficies digitalis nur eine Gelenksfacette (Fig. 4) und die S. dorsalis in einor dieser Art entsprechenden schmäleren Form, diesmal in Form eines hohen, nach unten sich verschmälernden, am Ende quer abgestutzten unregelmässigen Dreieckes (Fig. 3).

Es trägt an diesem, $8 \mathrm{~mm}$ breiten Ende einen beweglichen Anhang (b) vom Aussehen einer Epiphyse und der Gestalt eines Viertelsegmentes eines quer gelagerten ovalen Körpers.

Anhang und Lunaium sind durch eine Art straffer Gelenkkapsel $\left(\dagger^{\prime}, \dagger^{\prime}\right)$ mit einander vereinigt, in der beide Knochen an Flächen ohne Knorpelüberzüge an einander sich bewegen.

Der Anhang weist einen radialen stumpferen und einen ulnaren Pol, einen abgerundeten unteren Rand, eine Superficies superior s. brachialis, eine S. posterior s. dorsalis und eine S. anterior s. digitalis auf. Mit der S. brachialis bewegt sich der Anhang am unteren abgestutzten Ende der S. dorsalis des Lunatum; mit seiner rauhen S. dorsalis liegt er in der Verlängerung derselben Fläche des Lunatum (Fig. 3) mit der überknorpelten S. digitalis, in die sich die Gelenkfacette an der S. digitalis des Lunatum fortsetzt (Fig. 4), und articulirt an der ulnaren Facette der Gelenkfläche am Capitulum des Capitatum, ganz unten über der S. dorsalis des Körpers dieses Knochens.

Der Anhang war, abgesehen von der oben angegebenen Art straffer Gelenkkapsel zur Verbindung mit dem Lunatum, durch eine Faserschicht mit der Dorsalfläche des Körpers des Capitatum vereinigt.

Bedeutung. Der Anhang hat die Bedeutung einer anomal aufgetretenen persistirenden Epiphyse, in deren Synchondrose sich eine Art Gelenk entwickelt hat.

Wie das Lunatum bipartitum der 1. Art durch Auftreten 
einer anomalen Epiphyse in der volaren unteren Ecke des Lunatum und Entwickelung einer Art von Gelenk in der Synchondrose der Epiphyse bedingt war, so ist das Auftreten des L. bipartitum der 3. Art durch Auftreten einer anomalen Epiphyse im dorsalen unteren Ende des Lunatum und in der Bildung einer Art von Gelenk in der Synchondrose der Epiphyse begründet. Bei der 2. Art war das Lunatum in ein ganz grosses L. secundarium dorsale und in ein ganz kleines L. s. volare, bei der 3. Art aber ist es in ein ganz grosses L. secundarium volare und in ein ganz kleines L. s. dorsale getheilt. Nach den Embryologen ossificirt das knorplige Lunatum von einem Knochenkerne aus, nach Serres ${ }^{1}$ ) aber von 2 Knochenkernen. Wenn dieses Auftreten auch nur ein anomales ist, so giebt es doch Anhaltspunkte zu der Erklärung, „dass von den zwei Knochenkernen im knorpligen Lunatum 2 Stücke durch Bildungsanomalie besonders ossificiren, die so entstandenen 2 Knochenstücke durch Bildungshemmung isolirt persistiren und mit der Zeit durch Entwickelung eines Gelenkes in der Synchondrose beider mit einander beweglich verbunden werden können".

$\mathrm{Zu}$ bemerken ist, dass der Anhang des Lunatum dieses Falles (L. secundarium dorsale) ebenfalls im Centrum des Rückens des Carpus, und zwar auf dem Capitatum zwischen der Lücke für das Centrale carpi und der Lücke für das oben beschriebene neue supernumeräre Ossiculum carpi, Platz nimmt, also, wenn beide letzteren Knochen zugleich mit ihm vorhanden wären, zwischen diesen supernumerären Knochen, welche aus, vom Ursprunge an sicher oder doch höchst wahrscheinlich bestandenen Carpuselementen sich entwickelt haben, aber mit dem Lunatum in gar keiner oder doch nur in nebensächlicher Beziehung stehen ${ }^{2}$ ).

1) Bei Rambaud et Renault, Origine et développement des os. Paris 1864. $8^{\circ}$. p. 213.

2) Bei der 2. Art des Lunatum bipartitum habe ich am Knorpel der Superficies digitalis 2 Facetten, bei der 3. Art nur 1 Facette angetroffen. Im oben citirten Archiv f. Anatomie ete. Leipzig 1870. S. 493. Note 1. bemerkte ich: "Unter 220 Lunata (frischen) zeigte der Knorpel der Superficies digitalis an 106, also fast in der Hälfte der Fälle, 2 von einander deutlich geschiedene Facetten; in der an- 


\section{Erklärung der Abbildungen. \\ Taf. VIII. Fig. 3-4.}

Fig. 3. Ulnarstück des rechten Carpus von der Rückenseite.

Fig. 4. Lunatum von der nur mit einer Gelenkfacette versehenen Superficies digitalis bei aufwärts gestellter $\mathrm{S}$. dorsalis betrachtet. $1 \mathrm{Lu}-$ natum bipartitum. 2 Triquetrum. 3 Pisiforme. 4 Capitatum. 5 Hamatum. a Grosses Volarstück des Lunatum (L. secundarium volare). b Ganz kleines Dorsalstück oder Anhang (L. secundarium dorsale). + Art Gelenkkapsel zwischen beiden Stücken des Lunatum. ${ }^{\prime}$ Dieselbe am unteren vorderen Umfange geöffnet.

\section{III. (CXCV.) Ein im Centrum der Ulnarportion des Rückens des menschlichen Carpus zwischen dem Lunatum, Triquetrum und Hamatum gelagertes und articulirendes Ossiculum supernumerarium. Neue (6.) Art.}

(Hierzu Taf. VIII. Fig. 5-7.)

Die eine Art von Ueberzahl der Knochen im menschlichen Carpus ist durch Auftreten supernumerärer Knochen bedingt, die entweder schon rom Ursprunge an als nicht wesentliche Carpuselemente wirklich oder vermuthlich angelegt waren, oder als selbständige, zu articulirenden Knochen gewordene, ungewöhnliche Anhänge der Knochen der unteren Reihe sich erweisen, oder als selbständige und zu articulirenden Knochen gewordene Epiphysen der Metacarpalknochen zwischen die Knochen der unteren Reihen des Carpus sich ordneten und als Carpalknochen auftreten.

deren, etwas grösseren Hälfte 114 keine facettirte Fläche, oder doch nur eine Fläche mit einem feinen, schwach vertieften uharen Saume, der an macerirten Knochen nicht oder doch nur schwer zu erkennen ist." Ich habe somit durch Massenuntersuchungen nachgewiesen, dass das Vorkommen mit 2 Facetten an der $\mathrm{S}$. digitalis des frischen Lunatum zu dem Vorkommen mit nur 1 Facette sich verhält wie $53: 57$, oder in Procenten ausgedrückt, wie 47 oder 48 pCt.: 53 oder 52 pCt. Ich erwähne dies, damit man das Capital erfahre, von dem Wilhelm Krause (Handb. d. menschl. Anat. Bd. III. Hannover 1880. S. 77) für das Vorkommen der Lunata mit einer einfachen Facette am Knorpel der S. digitalis den Procentsatz von 52 pCt. annectirt hat. 